ORIGINAL RESEARCH

\title{
Chapter 2: Medical Tests Guidance (2) Developing the Topic and Structuring Systematic Reviews of Medical Tests: Utility of PICOTS, Analytic Frameworks, Decision Trees, and Other Frameworks
}

\author{
David Samson, $\mathrm{MS}^{\top}$ and Karen M. Schoelles, MD, SM, FACP \\ ${ }^{1}$ Technology Evaluation Center, Blue Cross and Blue Shield Association, Washington, DC, USA; ${ }^{2}$ ECRI Institute Health Technology Assessment \\ Group, ECRI Institute, Plymouth Meeting, PA, USA.
}

Topic development and structuring a systematic review of diagnostic tests are complementary processes. The goals of a medical test review are to identify and synthesize evidence to evaluate the impacts alternative testing strategies on health outcomes and to promote informed decisionmaking. A common challenge is that the request for a review may state the claim for the test ambiguously. Due to the indirect impact of medical tests on clinical outcomes, reviewers need to identify which intermediate outcomes link a medical test to improved clinical outcomes. In this paper, we propose the use of five principles to deal with challenges: the PICOTS typology (patient population, intervention, comparator, outcomes, timing, setting), analytic frameworks, simple decision trees, other organizing frameworks and rules for when diagnostic accuracy is sufficient.

KEY WORDS: diagnosis; review literature as topic; decision trees. J Gen Intern Med 27(Suppl 1):S11-9 DOI: $10.1007 / \mathrm{s} 11606-012-2007-7$

(C) The Author(s) 2012. This article is published with open access at Springerlink.com

"[We] have the ironic situation in which important and painstakingly developed knowledge often is applied haphazardly and anecdotally. Such a situation, which is not acceptable in the basic sciences or in drug therapy, also should not be acceptable in clinical applications of diagnostic technology."

J. Sanford (Sandy) Schwartz, Institute of Medicine, $1985^{1}$

Developing the topic creates the foundation and structure of an effective systematic review. This process includes understanding and clarifying a claim about a test (how a test might be of value in practice) and establishing the key questions to guide decisionmaking related to the claim. This typically involves specifying the clinical context in which the test might be used. Clinical context includes patient characteristics, how a new test might fit into existing diagnostic pathways, technical details of the test, characteristics of clinicians or operators using the test, management options and setting. Structuring the review refers to identifying the analytic strategy that will most directly achieve the goals of the review, accounting for idiosyncrasies of the data.

Topic development and structuring of the review are complementary processes. As evidence-based practice centers (EPCs) develop and refine the topic, the structure of the review should become clearer. Moreover, success at this stage reduces the chance of major changes in the scope of the review and minimizes rework. While this paper is intended to serve as a guide for EPCs, the processes described here are relevant to other systematic reviewers and a broad spectrum of stakeholders including patients, clinicians, caretakers, researchers, funders of research, government, employers, health care payers and industry, as well as the general public. This paper highlights challenges unique to systematic reviews of medical tests. For a general discussion of these issues as they exist in all systematic reviews, we refer the reader to previously published EPC methods papers ${ }^{2,3}$. This paper is one of 12 chapters in a JGIM and AHRQ supplement that address all aspects of preparation of systematic reviews of diagnostic tests.

\section{COMMON CHALLENGES}

The ultimate goal of a medical test review is to identify and synthesize evidence that will help evaluate the impacts on health outcomes of alternative testing strategies. Two common problems can impede achieving this goal. One is that the request for a review may state the claim for the test ambiguously. For example, a new medical test for Alzheimer's disease might fail to specify the patients who may benefit from the test-ranging from the use of the test as a screening tool among the "worried well" without evidence of deficit to using it as a diagnostic test in those with frank impairment and loss of function in daily living. Similarly, the request for a review of tests for prostate cancer might neglect to consider the role of such tests in clinical decisionmaking, such as guiding the decision to biopsy. 
Because of the indirect impact of medical tests on clinical outcomes, a second problem is how to identify which intermediate outcomes link a medical test to improved clinical outcomes compared to an existing test. The scientific literature related to the claim rarely includes direct evidence, such as randomized controlled trial results, in which patients are allocated to the relevant test strategies and evaluated for downstream health outcomes. More commonly, evidence about outcomes in support of the claim relates to intermediate outcomes, such as test accuracy.

\section{PRINCIPLES FOR ADDRESSING THE CHALLENGES Principle 1: Engage Stakeholders Using the PICOTS Typology}

In approaching topic development, reviewers should engage in a direct dialogue with the primary requestors and relevant users of the review (herein denoted "stakeholders") to understand the objectives of the review in practical terms; in particular, investigators should understand the sorts of decisions that the review is likely to affect. This serves to bring investigators and stakeholders to a shared understanding about the essential details of the tests and their relationship to existing test strategies (i.e., replacement, triage, or add-on), range of potential clinical utility, and potential adverse consequences of testing.

Operationally, the objective of the review is reflected in the key questions, which are normally presented in a preliminary form at the outset of a review. Reviewers should examine the proposed key questions to ensure that they accurately reflect the needs of stakeholders and are likely to be answered given the available time and resources. This is a process of trying to balance the importance of the topic against the feasibility of completing the review. Including a wide variety of stakeholders (such as the U.S. Food and Drug Administration [FDA], manufacturers, technical and clinical experts, and patients) can help provide additional perspectives on the claim and use of the tests. A preliminary examination of the literature can identify existing systematic reviews and clinical practice guidelines that may summarize evidence on current strategies for using the test and its potential benefits and harms.

The PICOTS typology (Patient population, Intervention, Comparator, Outcomes, Timing, Setting), defined in the Introduction to this Medical Test Methods Guide (Chapter 1 ), is a typology for defining particular contextual issues, and this formalism can be useful in focusing discussions with stakeholders. Furthermore, the PICOTS typology is a vital part of systematic reviews of both interventions and tests, lending them a transparent and explicit structure and influencing search methods, study selection and data extraction.

It is important to recognize that the process of topic refinement is iterative and PICOTS elements may change as the clinical context becomes clearer. Despite the best efforts of all participants, the topic may evolve even as the review is being conducted. Investigators should consider at the outset how such a situation will be addressed. ${ }^{4-6}$

\section{Principle 2: Develop an Analytic Framework}

We use the term "analytic framework" (sometimes called a causal pathway) to denote a specific form of graphical representation that specifies a path from the intervention or test of interest to all important health outcomes, including intervening steps and intermediate outcomes. ${ }^{7}$ Among PICOTS elements, the target patient population, intervention and clinical outcomes are specifically shown. The intervention can actually be viewed as a test and treat strategy as shown in links 2 through 5 . In the figure, the comparator is not shown explicitly, but is implied. Each linkage relating test, intervention, or outcome represents a potential key question and, it is hoped, a coherent body of literature.

The AHRQ EPC program has described the development and use of analytic frameworks in systematic reviews of interventions. Since the impact of tests on clinical outcomes usually depends on downstream interventions, analytic frameworks for systematic reviews of tests are particularly valuable and should be routinely included. The analytic framework is developed iteratively in consultation with stakeholders to illustrate and define the important clinical decisional dilemmas and thus serves to clarify important key questions further. ${ }^{2}$

However, systematic reviews of medical tests present unique challenge not encountered in reviews of therapeutic interventions. The analytic framework can help users to understand how the often-convoluted linkages between intermediate and clinical outcomes fit together, and to consider whether these downstream issues may be relevant to the review. Adding specific elements to the analytic framework will reflect the understanding gained about clinical context.

Harris and colleagues have described the value of the analytic framework in assessing screening tests for the U.S. Preventive Services Task Force (USPSTF). ${ }^{8}$ A prototypical analytic framework for medical tests as used by the USPSTF is shown in Figure 1. Each number in Figure 1 can be viewed as a separate key question that might be included in the evidence review.

In summarizing evidence, studies for each linkage might vary in strength of design, limitations of conduct, and 


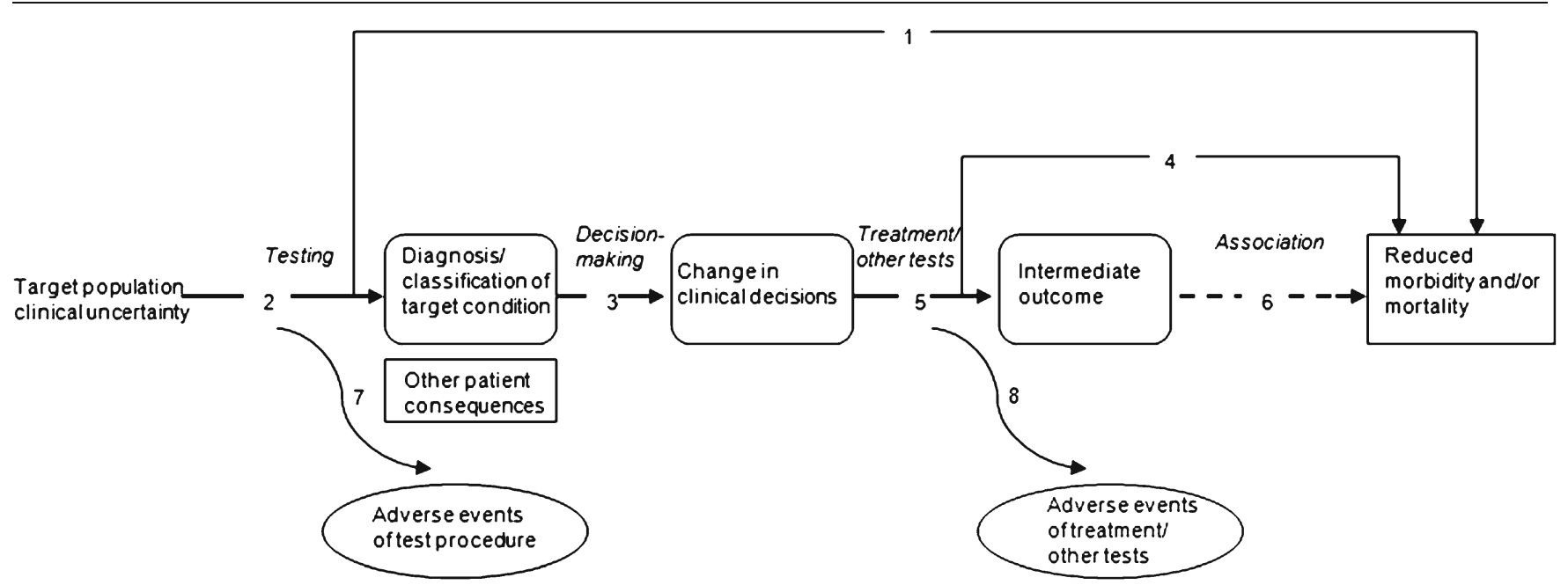

Research Questions

1. Direct evidence that testing reduces morbidity and/or mortality?

2. Test accuracy?

3. Impact of test on management?

4. Impact of management on health outcomes?

5. Impact of management on intermediate outcomes

6. Impact of intermediate outcomes on health outcomes

7. Adverse events, acceptability of test procedure?

8. Adverse events of subs equent treatment/other tests?

Figure 1. Application of USPSTF analytic framework to test evaluation. Adapted from Harris et al., $2001 .^{7}$

adequacy of reporting. The linkages leading from changes in patient management decisions to health outcomes are often of particular importance. The implication here is that the value of a test usually derives from its influence on some action taken in patient management. Although this is usually the case, sometimes the information alone from a test may have value independent of any action it may prompt. For example, information about prognosis that does not necessarily trigger any actions may have a meaningful psychological impact on patients and caregivers.

\section{Principle 3: Consider Using Decision Trees}

An analytic framework is helpful when direct evidence is lacking, showing relevant key questions along indirect pathways between the test and important clinical outcomes. Analytic frameworks are, however, not well-suited to depicting multiple alternative uses of the particular test (or its comparators) and are limited in their ability to represent the impact of test results on clinical decisions, and the specific potential outcome consequences of altered decisions. Reviewers can use simple decision trees or flow diagrams alongside the analytic framework to illustrate details of the potential impact of test results on management decisions and outcomes. Along with PICOTS specifications and analytic frameworks, these graphical tools represent systematic reviewers' understanding of the clinical context of the topic. Constructing decision trees may help to clarify key questions by identifying which indices of diagnostic accuracy and other statistics are relevant to the clinical problem and which range of possible pathways and outcomes (see Paper 3) practically and logically flow from a test strategy. Lord et al. describe how diagrams resembling decision trees define which steps and outcomes may differ with different test strategies, and thus the important questions to ask to compare tests according to whether the new test is a replacement, a triage, or an add-on to the existing test strategy. ${ }^{9}$

One example of the utility of decision trees comes from a review of noninvasive tests for carotid artery disease. ${ }^{10}$ In this review, investigators found that common metrics of sensitivity and specificity that counted both high-grade stenosis and complete occlusion as "positive" studies would not be reliable guides to actual test performance because the two results would be treated quite differently. This insight was subsequently incorporated into calculations of noninvasive carotid test performance. ${ }^{10,11}$ Additional examples are provided in the illustrations below. For further discussion on when to consider using decision trees, see Paper 10 in this series. 


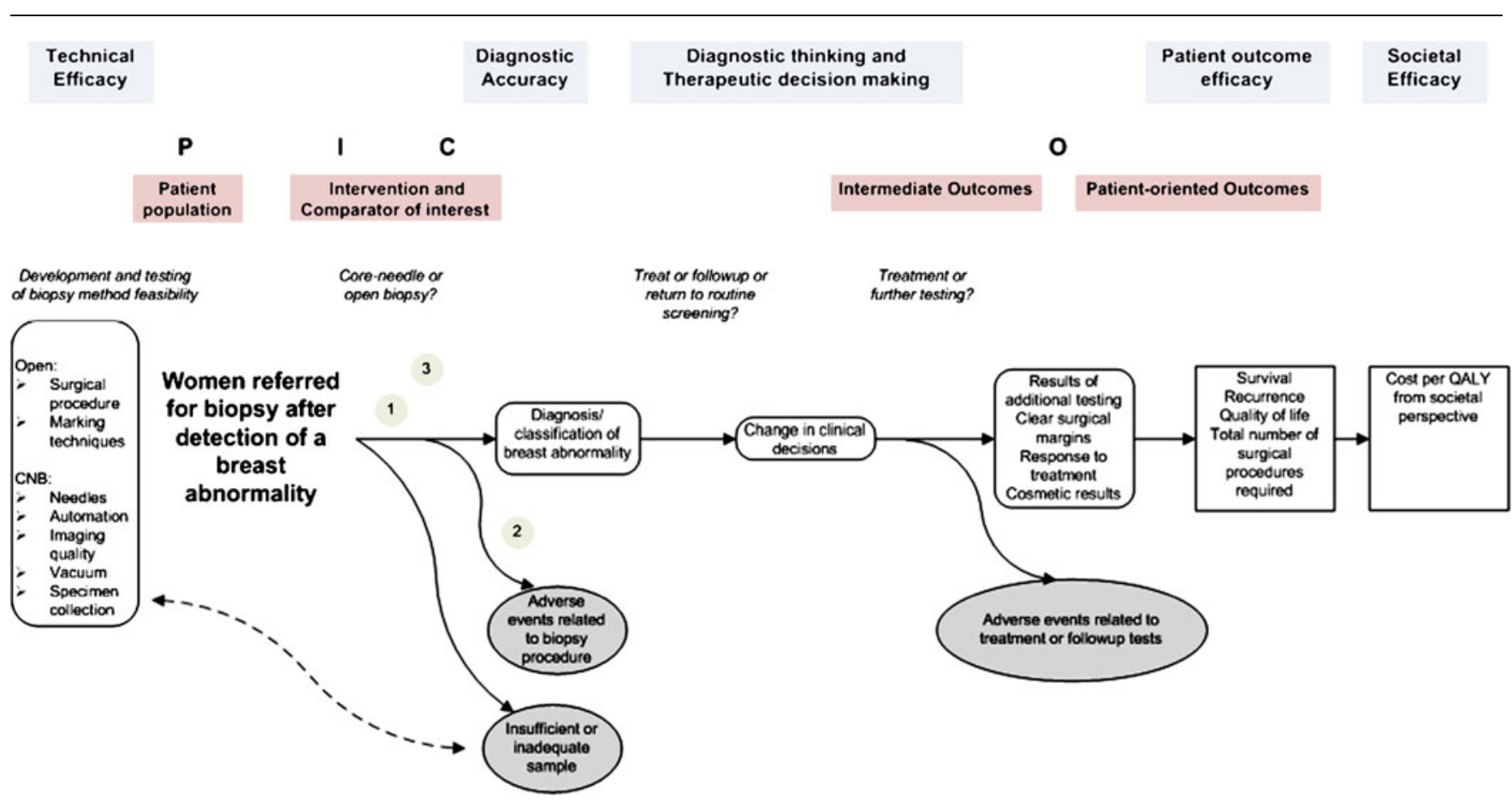

Figure 2. Example of an analytical framework within an overarching conceptual framework in the evaluation of breast biopsy techniques ${ }^{1}$. ${ }^{1}$ The numbers in the figure depict where the three key questions are located within the flow of the analytical framework.

\section{Principle 4: Sometimes it Is Sufficient to Focus Exclusively on Accuracy Studies}

Once reviewers have diagrammed the decision tree by which diagnostic accuracy may affect intermediate and clinical outcomes, it is possible to determine whether it is necessary to include key questions regarding outcomes beyond diagnostic accuracy. For example, diagnostic accuracy may be sufficient when the new test is as sensitive and as specific as the old test and the new test has advantages over the old test

Table 1. Examples of Initially Ambiguous Claims that were Clarified Through the Process of Topic Development

\begin{tabular}{|c|c|c|c|}
\hline & Full-Field Digital Mammography & HER2 & PET \\
\hline General topic & $\begin{array}{l}\text { FFDM to replace SFM in breast } \\
\text { cancer screening (Fig. 3) }\end{array}$ & $\begin{array}{l}\text { HER2 gene amplification assay as } \\
\text { add-on to HER2 protein expression } \\
\text { assay (Fig. 4) }\end{array}$ & PET as triage for breast biopsy (Fig. 5) \\
\hline $\begin{array}{l}\text { Initial ambiguous } \\
\text { claim }\end{array}$ & $\begin{array}{l}\text { FFDM may be a useful alternative to } \\
\text { SFM in screening for breast cancer }\end{array}$ & $\begin{array}{l}\text { HER2 gene amplification and protein } \\
\text { expression assays may complement } \\
\text { each other as means of selecting } \\
\text { patients for targeted therapy }\end{array}$ & $\begin{array}{l}\text { PET may play an adjunctive role to } \\
\text { breast examination and } \\
\text { mammography in detecting breast } \\
\text { cancer and selecting patients for } \\
\text { biopsy }\end{array}$ \\
\hline $\begin{array}{l}\text { Key concerns } \\
\text { suggested by } \\
\text { PICOTS, analytic } \\
\text { framework, and } \\
\text { decision tree }\end{array}$ & $\begin{array}{l}\text { Key statistics: sensitivity, diagnostic } \\
\text { yield, recall rate; similar types of } \\
\text { management decisions and outcomes } \\
\text { for index and comparator test-and- } \\
\text { treat strategies }\end{array}$ & $\begin{array}{l}\text { Key statistics: proportion of } \\
\text { individuals with intermediate/ } \\
\text { equivocal HER2 protein expression } \\
\text { results who have HER2 gene } \\
\text { amplification; key outcomes are } \\
\text { related to effectiveness of HER2- } \\
\text { targeted therapy in this subgroup }\end{array}$ & $\begin{array}{l}\text { Key statistics: negative predictive } \\
\text { value; key outcomes to be } \\
\text { contrasted were benefits of avoiding } \\
\text { biopsy versus harms of delaying } \\
\text { initiation of treatment for } \\
\text { undetected tumors }\end{array}$ \\
\hline Refined claim & $\begin{array}{l}\text { In screening for breast cancer, } \\
\text { interpretation of FFDM and SFM would } \\
\text { be similar, leading to similar } \\
\text { management decisions and outcomes; } \\
\text { FFDM may have a similar recall rate and } \\
\text { diagnostic yield at least as high as SFM; } \\
\text { FFDM images may be more expensive, } \\
\text { but easier to manipulate and store }\end{array}$ & $\begin{array}{l}\text { Among individuals with localized } \\
\text { breast cancer, some may have } \\
\text { equivocal results for HER2 protein } \\
\text { overexpression but have positive } \\
\text { HER2 gene amplification, identifying } \\
\text { them as patients who may benefit } \\
\text { from HER2-targeted therapy but } \\
\text { otherwise would have been missed }\end{array}$ & $\begin{array}{l}\text { Among patients with a palpable breast } \\
\text { mass or suspicious mammogram, if } \\
\text { FDG PET is performed before biopsy, } \\
\text { those with negative scans may avoid } \\
\text { the adverse events of biopsy with } \\
\text { potentially negligible risk of delayed } \\
\text { treatment for undetected tumor }\end{array}$ \\
\hline Reference & $\begin{array}{l}\text { Blue Cross and Blue Shield } \\
\text { Association Technology Evaluation } \\
\text { Center, } 2002^{15}\end{array}$ & Seidenfeld et al., $2008^{16}$ & Samson et al., $2002^{17}$ \\
\hline
\end{tabular}

Abbreviations: $F D G=$ fluorodeoxyglucose; FFDM = full-field digital mammography; HER2 = human epidermal growth factor receptor 2; PET = positron emission tomography; PICOTS = Patient population, Intervention, Comparator, Outcomes, Timing, Setting; SFM = screen-film mammography 
such as fewer adverse effects, is less invasive, is easier to use, provides results more quickly or is lower in cost. Implicit in this example is the comparability of downstream management decisions and outcomes between the test under evaluation and the comparator test. Another instance when a review may be limited to evaluation of sensitivity and specificity is when the new test is as sensitive as, but more specific than, the comparator, allowing avoidance of harms of further tests or unnecessary treatment. This situation requires the assumptions that the same cases would be detected by both tests and that treatment efficacy would be unaffected by which test was used. ${ }^{12,13}$

Particular questions to consider when reviewing analytic frameworks and decision trees to determine if diagnostic accuracy studies alone are adequate include:

1. Are extra cases detected by the new, more sensitive test similarly responsive to treatment as are those identified by the older test?
2. Are trials available that selected patients with the new test?

3. Do trials assess whether the new test results predict response?

4. If available trials selected only patients assessed with the old test, do extra cases identified with the new test represent the same spectrum or disease subtypes as trial participants?

5. Are tests' cases subsequently confirmed by same reference standard?

6. Does the new test change the definition or spectrum of disease (e.g., earlier stage)?

7. Is there heterogeneity of test accuracy and treatment effect (i.e., do accuracy and treatment effects vary sufficiently according to levels of a patient characteristic to change the comparison of the old and new test)?

When the clinical utility of an older comparator test has been established, and the first five questions can all be answered in the affirmative, then diagnostic accuracy evidence alone may be sufficient to support conclusions about a new test.

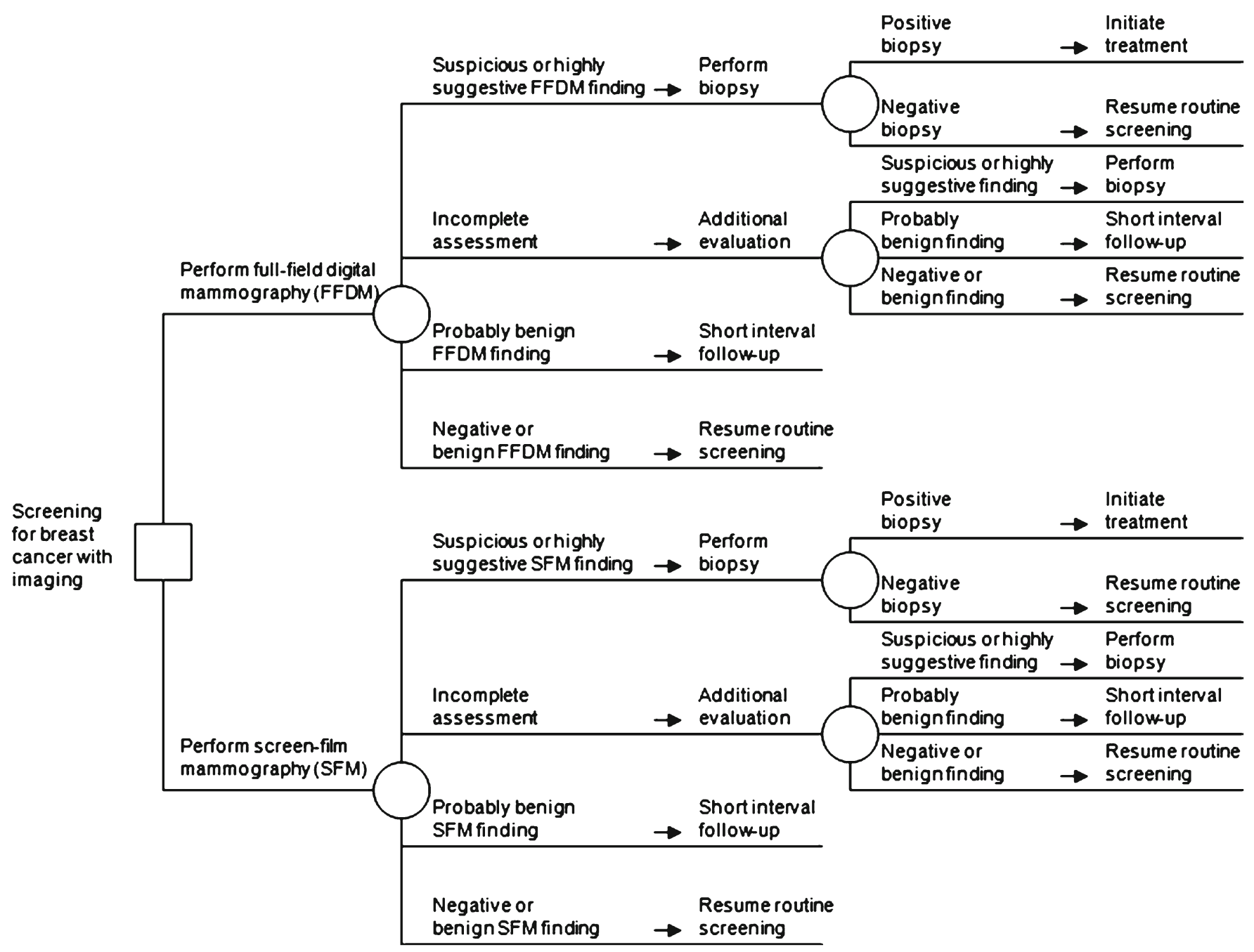

Figure 3. Replacement test example: full-field digital mammography versus screen-film mammography*. * Figure taken from Blue Cross and Blue Shield Association Technology Evaluation Center, 2002. 


\section{Principle 5: Other Frameworks May Be Helpful}

Various other frameworks (generally termed "organizing frameworks," as described briefly in the Introduction to this Medical Test Methods Guide [Paper 1]) relate to categorical features of medical tests and medical test studies. Lijmer and colleagues reviewed the different types of organizational frameworks and found 19 frameworks, which generally classify medical test research into 6 different domains or phases, including technical efficacy, diagnostic accuracy, diagnostic thinking efficacy, therapeutic efficacy, patient outcome, and societal aspects. ${ }^{13}$

These frameworks serve a variety of purposes. Some researchers, such as Van Den Bruel and colleagues, consider frameworks as a hierarchy and a model for how medical tests should be studied, with one level leading to the next (i.e., success at each level depends on success at the preceding level). ${ }^{14}$ Others, such as Lijmer and colleagues have argued that "The evaluation frameworks can be useful to distinguish between study types, but they cannot be seen as a necessary sequence of evaluations. The evaluation of tests is most likely not a linear but a cyclic and repetitive process." 13

We suggest that rather than being a hierarchy of evidence, organizational frameworks categorize key questions and suggest which types of studies would be most useful for the review. They may guide the clustering of studies; this may improve the readability of a review document. No specific framework is recommended, and indeed the categories of most organizational frameworks at least approximately line up with the analytic framework and the PICO(TS) elements as shown in Figure 2.

\section{Illustrations}

To illustrate the principles above, we describe three examples. In each case, the initial claim was at least somewhat ambiguous. Through the use of the PICOTS typology, the analytic framework, and simple decision trees, the systematic reviewers worked with stakeholders to clarify the objectives and analytic approach (Table 1). In addition to the examples described here, the AHRQ Effective Health Care Program website (http://effectivehealthcare.ahrq.gov/) offers free access to ongoing and completed reviews, containing specific applications of the PICOTS typology and analytic frameworks.

The first example concerns full-field digital mammography (FFDM) as a replacement for screen-film mammography (SFM) in screening for breast cancer; the review was conducted by the Blue Cross and Blue Shield Association Technology Evaluation Center. ${ }^{15}$ Specifying PICOTS elements and constructing an analytic framework were straightforward, with the latter resembling Figure 2 in form. In addition, with stakehoder input a simple decision tree was drawn (Fig. 3) which revealed that the management

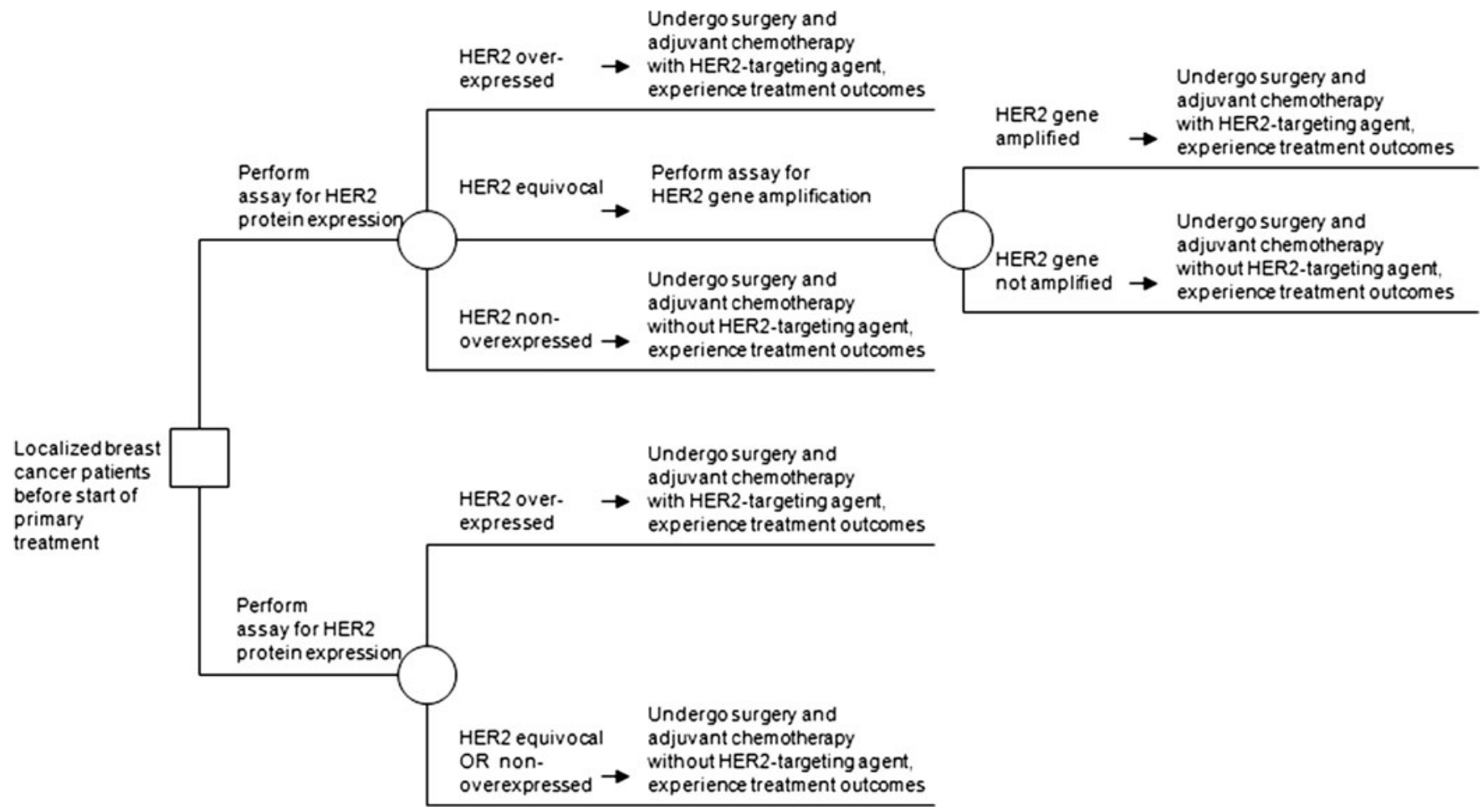

Figure 4. Add-on test example: HER2 protein expression assay followed by HER2 gene amplification assay to select patients for HER2targeted therapy*. Abbreviation: HER2 = human epidermal growth factor receptor 2. * Figure taken from Seidenfeld et al., $2008 .{ }^{15}$ 
decisions for both screening strategies were similar, thus downstream treatment outcomes were not a critical issue. The decision tree also showed that the key indices of test performance were sensitivity, diagnostic yield, and recall rate. These insights were useful as the project moved to abstracting and synthesizing the evidence, which focused on accuracy and recall rates. In this example, the reviewers concluded that FFDM and SFM had comparable accuracy and led to comparable outcomes; however, storing and manipulating images was much easier for FFDM than for SFM.

The second example concerns use of the human epidermal growth factor receptor 2 (HER2) gene amplification assay after the HER2 protein expression assay to select patients for HER2-targeting agents as part of adjuvant therapy among patients with localized breast cancer. ${ }^{16}$ The HER2 gene amplification assay has been promoted as an add-on to the HER2 protein expression assay. Specifically, individuals with equivocal HER2 protein expression would be tested for amplified HER2 gene levels; in addition to those with increased HER2 protein expression, patients with elevated levels by amplification assay would also receive adjuvant chemotherapy that includes HER2-targeting agents. Again, PICOTS and an analytic framework were developed, establishing the basic key questions. In addition, the authors constructed a decision tree (Fig. 4) that made it clear that the treatment outcomes affected by HER2 protein and gene assays were at least as important as the test accuracy. While in the first case, the reference standard was actual diagnosis by biopsy, here the reference standard is the amplification assay itself. The decision tree identified the key accuracy index as the proportion of individuals with equivocal HER2 protein expression results who have positive amplified HER2 gene assay results. The tree exercise also indicated that one key question must be whether HER2-targeted therapy is effective for patients who had equivocal results on the protein assay but were subsequently found to have positive amplified HER2 gene assay results.

The third example concerns use of fluorodeoxyglucose positron emission tomography (FDG PET) as a guide to the decision to perform a breast biopsy on a patient with either

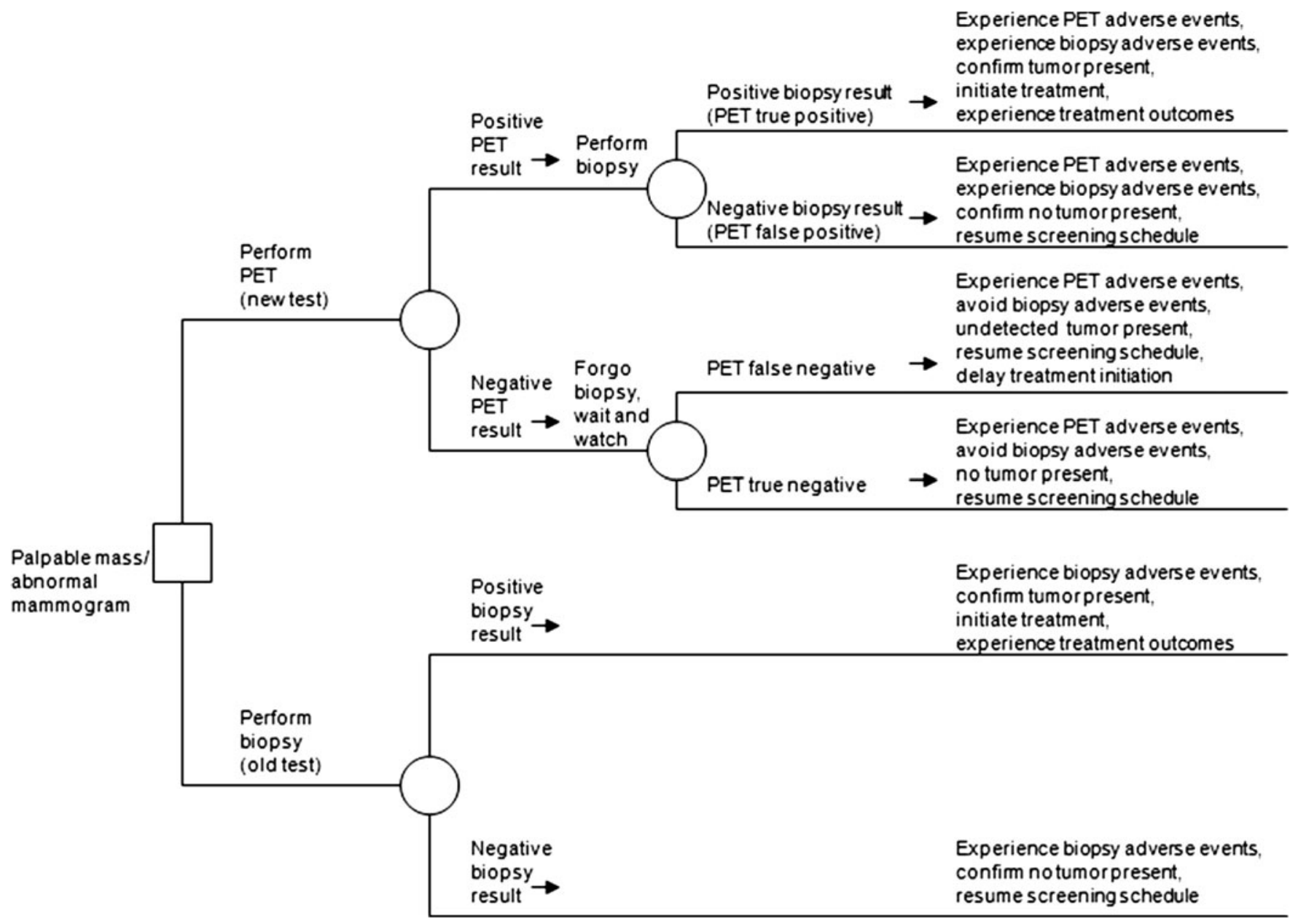

Figure 5. Triage test example: positron emission tomography (PET) to decide whether to perform breast biopsy among patients with a palpable mass or abnormal mammogram*. * Figure taken from Samson et al., 2002. ${ }^{17}$ 
a palpable mass or an abnormal mammogram. ${ }^{17}$ Only patients with a positive PET scan would be referred for biopsy. Table 1 shows the initial ambiguous claim, lacking PICOTS specifications such as the way in which testing would be done. The analytic framework was of limited value as several possible relevant testing strategies were not represented explicitly in an analytic framework. The authors constructed a decision tree (Fig. 5). The testing strategy in the lower portion of the decision tree entails performing biopsy in all patients, while the triage strategy uses a positive PET finding to rule in a biopsy and a negative PET finding to rule out a biopsy. The decision tree illustrates that the key accuracy index is negative predictive value: the proportion of negative PET results that are truly negative. The tree also reveals that the key contrast in outcomes involves any harms of delaying treatment for undetected cancer when PET is falsely negative versus the benefits of safely avoiding adverse effects of the biopsy when PET is truly negative. The authors concluded that there is no net beneficial impact on outcomes when PET is used as a triage test to select patients for biopsy among those with a palpable breast mass or suspicious mammogram. Thus, estimates of negative predictive values suggest that there is an unfavorable trade-off between avoiding the adverse effects of biopsy and delaying treatment of an undetected cancer.

This case illustrates when a more formal decision analysis may be useful, specifically when new test has higher sensitivity but lower specificity than the old test, or vice versa. Such a situation entails tradeoffs in relative frequencies of true positives, false negatives, false positives, and true negatives, which decision analysis may help to quantify.

\section{SUMMARY}

The immediate goal of a systematic review of a medical test is to determine the health impacts of use of the test in a particular context or set of contexts relative to one or more alternative strategies. The ultimate goal is to produce a review that promotes informed decisionmaking.

Key points are:

- Reaching the above-stated goals requires an interactive and iterative process of topic development and refinement aimed at understanding and clarifying the claim for a test. This work should be done in conjunction with the principal users of the review, experts, and other stakeholders.

- The PICOTS typology, analytic framework, simple decision trees, and other organizing frameworks are all tools that can minimize ambiguity, help identify where review resources should be focused, and guide the presentation of results.

- Sometimes it is sufficient to focus only on accuracy studies. For example, diagnostic accuracy may be sufficient when the new test is as sensitive and specific as the old test and the new test has advantages over the old test such as fewer adverse effects, is less invasive, is easier to use, provides results more quickly or is lower in cost.

Acknowledgements: We wish to thank David Matchar and Stephanie Chang for their valuable contributions. Work on this paper was funded by AHRQ.

Conflict of Interest: The authors declare that they do not have a conflict of interest.

Open Access: This article is distributed under the terms of the Creative Commons Attribution License which permits any use, distribution, and reproduction in any medium, provided the original author(s) and the source are credited.

Corresponding Author: David Samson, MS; Technology Evaluation Center, Blue Cross and Blue Shield Association, 1310 G Street, NW, Washington, DC 20005, USA (e-mail: David.samson@bcbsa. com).

\section{REFERENCES}

1. Institute of Medicine, Division of Health Sciences Policy, Division of Health Promotion and Disease Prevention, Committee for Evaluating Medical Technologies in Clinical Use. Assessing medical technologies. Washington, DC: National Academy Press; 1985. Chapter 3: Methods of technology assessment. p. 80-90.

2. Helfand M, Balshem H. AHRQ Series Paper 2: Principles for developing guidance: AHRQ and the Effective Health-Care Program. J Clin Epidemiol. 2010;63(5):484-90.

3. Whitlock EP, Lopez SA, Chang S, et al. AHRQ Series Paper 3: Identifying, selecting, and refining topics for comparative effectiveness systematic reviews: AHRQ and the Effective Health-Care program. J Clin Epidemiol. 2010;63(5):491-501.

4. Matchar DB, Patwardhan M, Sarria-Santamera A, et al. Developing a Methodology for Establishing a Statement of Work for a Policy-Relevant Technical Analysis. Technical Review 11. (Prepared by the Duke Evidence-based Practice Center under Contract No. 290-02-0025.) AHRQ Publication No. 06-0026. Rockville, MD: Agency for Healthcare Research and Quality. January 2006. Available at: http://www.ahrq.gov/downloads/pub/evidence/pdf/statework/statework.pdf. Accessed January $10,2012$.

5. Sarria-Santamera A, Matchar DB, Westermann-Clark EV, et al. Evidence-based practice center network and health technology assessment in the United States: bridging the cultural gap. Int $\mathrm{J}$ Technol Assess Health Care. 2006;22(1):33-8.

6. Patwardhan MB, Sarria-Santamera A, Matchar DB, et al. Improving the process of developing technical reports for health care decision makers: using the theory of constraints in the evidence-based practice centers. Int J Technol Assess Health Care. 2006;22(1):26-32.

7. Woolf SH. An organized analytic framework for practice guideline development: using the analytic logic as a guide for reviewing evidence, developing recommendations, and explaining the rationale. In: McCormick KA, Moore SR, Siegel RA, eds. Methodology perspectives: clinical practice guideline development. Rockville, MD: U.S. Department of Health and Human Services, Public Health Service, Agency for Health Care Policy and Research; 1994:105-13.

8. Harris RP, Helfand M, Woolf $\mathbf{~ S H}$, et al. Current methods of the US Preventive Services Task Force: a review of the process. Am J Prev Med. 2001;20(3 Suppl):21-35.

9. Lord SJ, Irwig L, Bossuyt PM. Using the principles of randomized controlled trial design to guide test evaluation. Med Decis Making. 2009;29(5):E1-E12. Epub2009 Sep 22.

10. Feussner JR, Matchar DB. When and how to study the carotid arteries. Ann Intern Med. 1988;109(10):805-18. 
11. Blakeley DD, Oddone EZ, Hasselblad V, et al. Noninvasive carotid artery testing. A meta-analytic review. Ann Intern Med. 1995;122(5):360-7.

12. Lord S J, Irwig $\mathbf{L}$, Simes $\mathbf{J}$. When is measuring sensitivity and specificity sufficient to evaluate a diagnostic test, and when do we need a randomized trial? Ann Intern Med. 2006;144(11):850-5.

13. Lijmer JG, Leeflang M, Bossuyt PM. Proposals for a phased evaluation of medical tests. Med Decis Making. 2009;29(5):E13-21.

14. Van den Bruel A, Cleemput I, Aertgeerts B, et al. The evaluation of diagnostic tests: evidence on technical and diagnostic accuracy, impact on patient outcome and cost-effectiveness is needed. $\mathrm{J}$ Clin Epidemiol. 2007;60(11):1116-22.

15. Blue Cross and Blue Shield Association Technology Evaluation Center (BCBSA TEC). Full-field digital mammography. Volume 17, Number 7, July 2002.
16. Seidenfeld J, Samson DJ, Rothenberg BM, et al. HER2 Testing to Manage Patients With Breast Cancer or Other Solid Tumors. Evidence Report/Technology Assessment No. 172. (Prepared by Blue Cross and Blue Shield Association Technology Evaluation Center Evidence-based Practice Center, under Contract No. 290-02-0026.) AHRQ Publication No. 09-E001. Rockville, MD: Agency for Healthcare Research and Quality. November 2008. Available at: www.ahrq.gov/ downloads/pub/evidence/pdf/her2/her2.pdf. Accessed January 10, 2012.

17. Samson DJ, Flamm CR, Pisano ED, et al. Should FDG PET be used to decide whether a patient with an abnormal mammogram or breast finding at physical examination should undergo biopsy? Acad Radiol. 2002:9(7):773-83. 